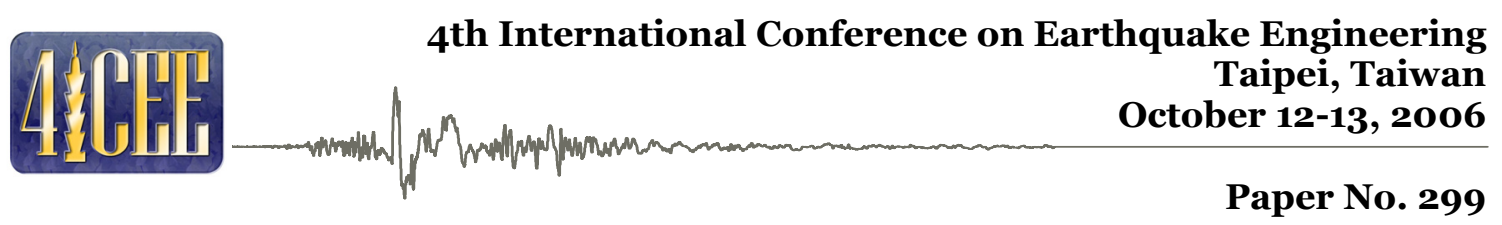

\title{
REAL-TIME HYBRID TESTINg USING MODEL-BASED DELAY COMPENSATION
}

\author{
Juan E. Carrion ${ }^{1}$ and B.F. Spencer $\mathrm{Jr}^{2}$
}

\begin{abstract}
Real-time hybrid testing is an attractive method to evaluate the response of structures under earthquake loads. The method is a variation of the pseudodynamic testing technique in which the simulation is executed in real time, thus allowing investigation of structural systems with timedependent components. Real-time hybrid testing is challenging because it requires performance of all the calculations, application of the displacements, and acquisition of the measured forces, within a very small time frame (i.e., a single time step). Furthermore, unless appropriate compensation for time delays and actuator time lag is implemented, stability problems are likely to occur during the experiment (especially during testing of structures with high natural frequencies, e.g., stiff structures or multi-degree-of-freedom systems). This paper presents an approach for real-time hybrid testing in which time delay/lag compensation is implemented using model-based response prediction. The efficacy of the proposed strategy is verified by conducting substructure real-time hybrid testing of a steel frame under earthquake loads. Experimental results agree well with the analytical solution and show that the approach is capable of achieving a time-scale expansion factor of one (i.e., real time). Additionally, the proposed method allows testing structures with larger frequencies than when using the conventional polynomial extrapolation method, thus extending the capabilities of the real-time hybrid testing technique.
\end{abstract}

Keywords: Real-time hybrid testing, experimental testing, delay compensation, substructuring.

\section{INTRODUCTION}

Experimental testing is an essential tool for understanding how structures respond to extreme events such as earthquakes. Hybrid (or pseudodynamic) testing provides an attractive alternative for dynamic testing of structural systems by combining physical testing with numerical simulation (Takanashi et al. 1975). The basis of the method is that the dynamic response of the structure due to a particular loading (e.g., earthquake) is calculated numerically on a computer and then the restoring forces from the structure are obtained by applying the calculated displacements to the physical test structure. The structure to be tested is divided into a physical component and a numerical model. In its basic form, the numerical model includes the mass on the structure (lumped at discrete locations), and the structural damping. During the test, the response of the structure is calculated using time stepintegration of the equation of motion. The displacements obtained are then imposed to the physical structure using actuators at the discrete locations where lumped masses are assumed. The forces required to produce these displacements are measured and fed back to the computer to calculate the

\footnotetext{
${ }^{1} \mathrm{Ph} . \mathrm{D}$. Candidate, Department of Civil and Environmental Engineering, University of Illinois at Urbana-Champaign, Urbana, Illinois 61801, U.S.A., jcarrion@uiuc.edu

${ }^{2}$ Professor, Department of Civil and Environmental Engineering, University of Illinois at Urbana-Champaign, Urbana, Illinois 61801, U.S.A., bfs@uiuc.edu
} 
displacements corresponding to the next time step. When not required or desired to test the entire structure, the substructure technique allows physical testing of only the parts of the structure of interest (e.g., where damage is expected, or components that are difficult to model numerically), while the rest of the structure can be modeled numerically. In conventional hybrid testing, the displacements are imposed on an extended time-scale which typically ranges from 100 to 1000 times the actual earthquake duration. Therefore, when the restoring forces depend also on the velocity (i.e., ratedependent), the method is not applicable.

Real-time hybrid testing is a variation of the pseudodynamic testing method in which the imposed displacements and response analysis are executed in a common time scale (i.e., real time), thus allowing testing of systems with rate-dependent components (Nakashima et al. 1992; Horiuchi et al. 1996; Horiuchi et al. 1999; Nakashima and Masaoka 1999; Darby et al. 2001; Shing et al. 2004). Realtime hybrid testing makes it possible to test the large category of structural components associated with vibration control including passive, semi-active, and active control devices (e.g., base isolation and dampers), which are typically nonlinear and rate-dependent. Real-time hybrid testing is challenging because it is necessary to perform all the calculations, apply the displacements, and measure and feedback the forces within a single time step (typically less than $20 \mathrm{~ms}$ ). Because the test is conducted in real time, the dynamics of the testing system and specimen become important. Furthermore, when hydraulic actuators are used to apply forces to the test specimen there is a time lag between the time at which the displacement is commanded and the time when the actuator actually reaches the desired position. There are also some inevitable time delays associated with the numerical calculations and the communication between computer and data acquisition systems. Because of these time delays and lags, the force measured and fed back from the experiment does not correspond to the desired position (it is measured before the actuator has reached its target position). The effect of this error is to introduce additional energy into the system which may even cause the test to go unstable (Horiuchi et al. 1996).

This paper presents an approach for real-time hybrid testing which implements model-based time delay/lag compensation. The efficacy of the proposed strategy is verified by conducting substructure real-time hybrid testing of a steel frame under earthquake loads in which the physical substructure is a small-scale specimen representing one of the columns. The performance of the testing system and compensation technique is investigated by considering structures with different fundamental frequencies. The proposed time delay/lag compensation technique is also compared to the traditionally used polynomial extrapolation method.

\section{REAL-TIME HYBRID TESTING}

The equation of motion of the structural system to be tested is given by the following second-order differential equation,

$$
\mathbf{M} \ddot{\mathbf{x}}(t)+\mathbf{C} \dot{\mathbf{x}}(t)+\mathbf{R}(\mathbf{x}, \dot{\mathbf{x}}, t)=\mathbf{F}(t)
$$

in which $\mathbf{M}$ is the mass matrix, $\mathbf{C}$ the damping matrix (for linear damping), $\mathbf{R}$ the restoring force vector, $\mathbf{F}$ the vector of externally applied forces, and $\mathbf{x}$ the displacement vector. The dots denote differentiation with respect to time. The equation of motion is discretized by dividing the time interval at a uniform spacing of $\Delta t$, and then solved using a step-by-step time integration algorithm. Amongst all the numerical algorithms proposed for hybrid testing, the central difference method (CDM) and the alpha operator-splitting method $\alpha$-OS (Nakashima et al., 1990) are the most widely used and are the ones considered herein. The expression to calculate displacements using the CDM is given by

$$
\mathbf{x}_{i+1}=\left[\frac{1}{\Delta t^{2}} \mathbf{M}+\frac{1}{2 \Delta t} \mathbf{C}\right]^{-1}\left[\frac{2}{\Delta t^{2}} \mathbf{M} \mathbf{x}_{i}-\left(\frac{1}{\Delta t^{2}} \mathbf{M}-\frac{1}{2 \Delta t} \mathbf{C}\right) \mathbf{x}_{i-1}-\mathbf{R}\left(\mathbf{x}_{i}\right)+\mathbf{F}_{i}\right]
$$


in which the subscript $i$ denotes the value of the variable at time $t_{i}=i \Delta t$. The advantage of the CDM is that it is an explicit method; therefore it requires only information from the current and previous steps to compute the response at the next time step, making its application to hybrid testing straightforward. However, the method is conditionally stable (with stability criterion given by $\omega \Delta t \leq 2$, where $\omega$ is the highest natural frequency of the system). For structures in which the stability criterion of the CDM becomes difficult to assure, the $\alpha$-OS-method, which is unconditionally stable for systems of softening type, is used.

\section{Actuator dynamics}

Displacements in hybrid testing are generally imposed on the structure using hydraulic actuators. When displacements are applied at fast rates, the dynamics of the actuator and attached specimen become important. Dyke et al. (1995) analyzed the effects of control-structure-interaction (CSI) and showed that the dynamics of the actuator and structure are directly linked (through a natural velocity feedback). This work demonstrated that neglecting phase differences between the command input and the resulting force, (i.e., neglecting the CSI), resulted in an apparent time delay associated in the literature with generation of the control forces. In structural testing, this effect is not significant when the tests are conducted at slow speeds. However, when conducting fast and real-time hybrid testing, this dynamic coupling and the finite response time of the hydraulic actuators become especially important, resulting in a time lag between the commanded displacement and the realization of this command by the actuator. Although the time lag is caused by both actuator dynamics and the attached test specimen, the contribution from the former is dominant (Zhao et al. 2003). Typically the natural frequency of the test specimen is large compared to the bandwidth of interest and in the pseudo-static region (low frequency region), the phase of the actuator transfer function can be approximated to be linear, resulting in a pure time delay. Actuator time lags can be several times larger than the typical time-step used for seismic testing. Typical values reported in the literature range from 8 to $30 \mathrm{~ms}$ (Horiuchi et al. 1999; Darby et al. 2001; Shing et al. 2004; Nakashima and Masaoka 1999).

In real-time hybrid testing, the effect of time delays (denoted here by $\delta t_{d}$ ) is that the output or imposed displacement lags the input or desired calculated displacement by an amount of time equal to the delay. Because of this delay, the force measured and fed back from the experiment does not correspond to the desired position (it is measured before the actuator has reached its target position), however the algorithm assumes that it corresponds to the desired calculated displacement. For a linear-elastic system the resulting response, as seen by the algorithm is a counter-clockwise hysteretic loop, instead of the straight line corresponding to the linear behavior. The effect of this counter-clockwise loop is to introduce additional energy into the simulation. Horiuchi et al. (1996) demonstrated that for a linearelastic SDOF system the increase in the total energy caused by the delay/lag is equivalent to introducing negative damping (given by $c_{e q}=-k \delta t_{d}$, where $k$ is the stiffness of the system). This artificial negative damping becomes large when either the stiffness of the system or the time delay/lag is large. When this negative damping is larger than the structural damping, the response will diverge (become unstable), and the experiment must be halted. Instability almost invariably occurs in practice due to the low levels of damping associated with structural frames and the large time delays/lags (Darby et al. 2001). Therefore, introduction of a compensation for time delays/lags is essential when conducting a fast hybrid experiment.

\section{Delay compensation}

Several techniques have been proposed to compensate for time delays and actuator lag, of which the response prediction method (Horiuchi et al. 1996) has been the most widely used. In this approach, instead of using the calculated displacement as the command signal to the actuator, the displacement of the actuator after the delay $\delta t_{d}$ is predicted and used as the command signal to the actuator. Because of the time delay, the resulting displacement imposed by the actuator approximates the calculated one. This approach is illustrated in Figure 1(a), where $x\left(t_{i+1}\right)$ is the calculated displacement, $\hat{x}\left(t_{i+1}+\delta t_{d}\right)$ is the predicted (compensated) displacement, and $x^{m}\left(t_{i+1}\right)$ is the actual displacement imposed by the actuator. 

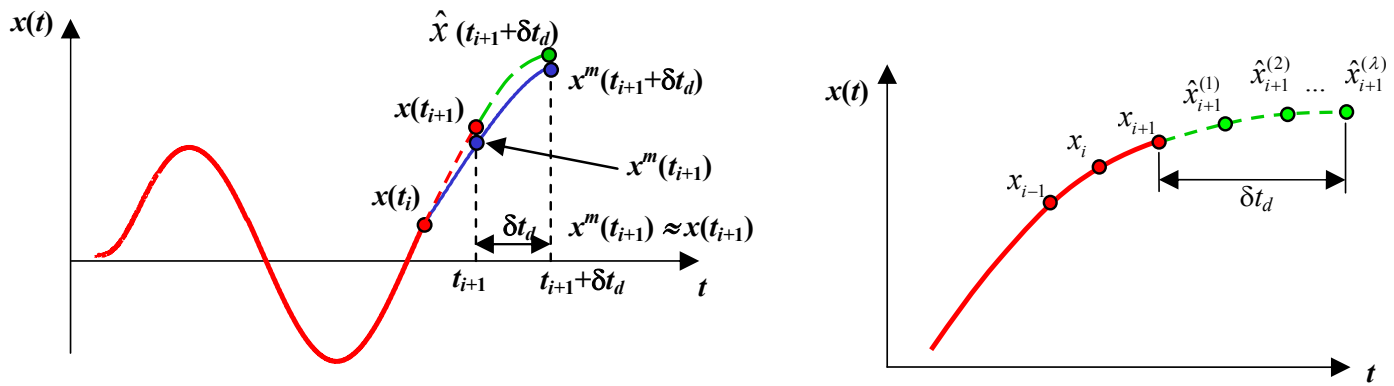

Figure 1. (a) Delay compensation using response prediction, (b) Calculation of predicted displacements using model-based response prediction.

The method used for the prediction by Horiuchi et al. (1996) was an $n$-th order polynomial extrapolation based on the displacements at the present and previous time steps. The accuracy and stability of the method were investigated using a linear single-degree-of-freedom (SDOF) system. The prediction method was found to cause variations in both stiffness and damping. Furthermore, damping becomes negative and the simulation diverges when the non-dimensional parameter $\omega \delta t_{d}$ (where $\omega$ is the natural frequency of the system) is larger than a critical value that depends on the order of the polynomial used for the extrapolation. For multi-degree-of-freedom systems, the critical value should be based on the highest natural frequency of the structure (Horiuchi et al. 1999). A third-order polynomial extrapolation was recommended because it requires a small calculation time and gives a relatively large critical value $\omega \delta t_{d}=1.571$. The limitation imposed by the critical value for stability becomes very important for high frequencies corresponding to stiff structures or multi-degree-offreedom systems, or when the delays/lags are large compared to the smallest period of the structure.

\section{MODEL-BASED RESPONSE PREDICTION}

The polynomial extrapolation method for response prediction uses only information from the displacements at a few previous time steps. For small prediction times, the response of a structure (which is nearly harmonic) can be well represented by polynomials, but when longer prediction times are required, or for systems with larger natural frequencies (i.e., shorter natural periods), more precise prediction methods are necessary. A model-based response prediction method accounts for the physical characteristics of the response by incorporating known information about the system. Although the complete characteristics of the system are not known in advance, there is some information about the system that is known prior to testing or can be calculated at the beginning of the experiment. This information includes: the mass matrix $\mathbf{M}$, the damping matrix $\mathbf{C}$, the external excitation $\mathbf{F}$ (e.g., ground acceleration $\ddot{\mathbf{x}}_{g}$ for seismic loading), and the initial elastic stiffness of the structure $\mathbf{K}_{\mathrm{e}}$. By incorporating known information about the system and the excitation, a more accurate prediction of the response can be achieved, allowing larger prediction horizons, stability improvements, and testing of structures with higher natural frequencies or with more degrees-offreedom.

The proposed model-based response predictor uses the information available at the beginning of each time step to predict the displacement response at the end of the prediction horizon (i.e., the time delay). The prediction is performed using a model of the system with the uncompensated target displacement $x_{i+1}$ as initial condition. The response of the predictor model is then computed for the displacements $\hat{x}_{i+1}^{(k)}$ for $k=1,2, \ldots, \lambda$, where $\lambda$ is the ratio between the time delay and the time step (i.e., $\lambda=\delta t_{d} / \Delta t$ ), as shown on Figure 1(b) (the time step size $\Delta t$ is set so that the time delay is an integer multiple of $\Delta t$ for simplicity). The predicted displacement at the end of the prediction horizon $\left(\hat{x}_{i+1}^{(\lambda)}\right)$ is then sent as a command to the actuator, providing the compensation for the time delay.

To predict the response of structural systems with a small number of degrees-of-freedom, a complete model of the system is used to generate the predictions. By considering the solution of the equation of 
motion (e.g., Eq. 2 when using the Central Difference Method), the only unknown parameter is the restoring force vector $\mathbf{R}$, while the mass matrix, damping matrix, and external force vector are known. An approximation of the restoring force vector can be obtained using the previous measured restoring force vector and a certain stiffness matrix $\mathbf{K}$ as given by

$$
\mathbf{R}_{i+1} \approx \mathbf{R}_{i}+\mathbf{K}\left(\mathbf{x}_{i+1}-\mathbf{x}_{i}\right)
$$

When the stiffness of the system does not change significantly during the experiment (e.g., linear and moderately nonlinear response), using the initial elastic stiffness of the system on the approximation (i.e., $\mathbf{K}=\mathbf{K}_{\mathbf{e}}$ ) yields good estimates of the restoring force (a similar approximation is used in the Operator-Splitting method by Nakashima et al. 1990). When the response of the structure is limited to the elastic range, this approximation and the proposed model-based compensation method yields the exact prediction, and theoretically the experiment should be stable for any value of the parameter $\omega \delta t_{d}$.

For cases when the stiffness of the system changes significantly during the experiment, an estimate of the tangent stiffness based on the last increment of the measured restoring force is used. A simple estimate of the tangent stiffness can be obtained using the Broyden formula (Broyden 1965) which corresponds to a generalization of the one-dimensional secant approximation to the tangent stiffness. The tangent stiffness $\mathbf{K}_{i}$ is obtained by making the least change to $\mathbf{K}_{i-1}$ that satisfies the equation $\mathbf{K}_{i} \Delta \mathbf{x}_{i}=\Delta \mathbf{r}_{i}$, where $\Delta \mathbf{x}_{i}=\mathbf{x}_{i}-\mathbf{x}_{i-1}$ and $\Delta \mathbf{r}_{i}=\mathbf{R}_{i}-\mathbf{R}_{i-1}$ are the displacement and force increments, respectively. The equation for the stiffness using the Broyden formula is

$$
\mathbf{K}_{i}=\mathbf{K}_{i-1}+\frac{\left(\Delta \mathbf{r}_{i}-\mathbf{K}_{i-1} \Delta \mathbf{x}_{i}\right) \Delta \mathbf{x}_{i}^{T}}{\Delta \mathbf{x}_{i}^{T} \Delta \mathbf{x}_{i}}
$$

For systems with a large number of degrees-of-freedom, using a complete model of the system to conduct the response prediction is not efficient. Considering that the earthquake response of typical structures is dominated by a few lower modes, the mode superposition technique (based on the elastic mode shapes) is used to predict the response of larger systems.

The above formulation provides a simple and efficient method for model-based response prediction which gives good predictions of the response even for systems with inelastic response. The next section describes the experimental setup used to validate the proposed approach.

\section{EXPERIMENTAL SETUP}

A system that combines fast hardware, for high-speed computations and communication, with high performance hydraulic components has been employed to experimentally validate the proposed realtime hybrid testing approach (see Figure 2). The system consists of a hydraulic actuator controlled by a digital servo-controller, a displacement transducer which measures the displacements imposed by the actuator and provides position feedback, a load cell to measure the force imposed by the actuator (which corresponds to the restoring force from the specimen), a computer with a board for real-time control (to solve the equation of motion and generate the target displacements), and digital to analog and analog to digital converters. 


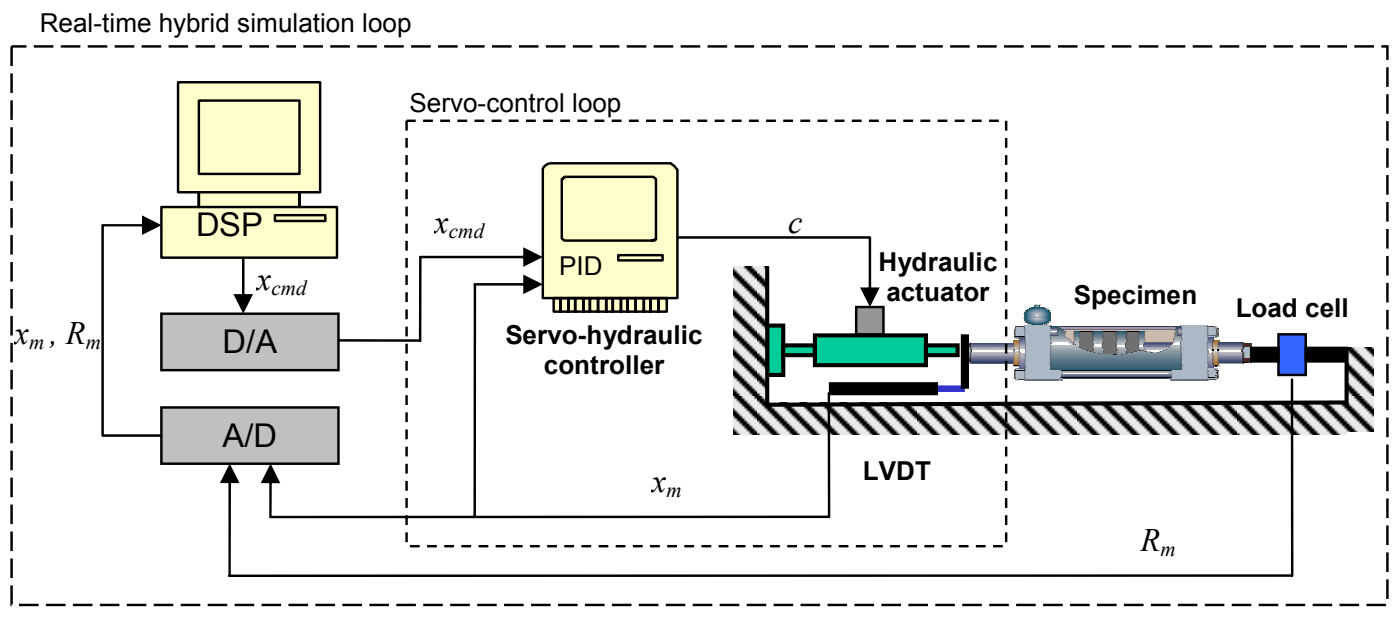

Figure 2. Schematic of experimental setup ( $x_{m}=$ measured displacement, $R_{m}=$ measured restoring force, $x_{c m d}=$ command displacement, and $c=$ command to the actuator).

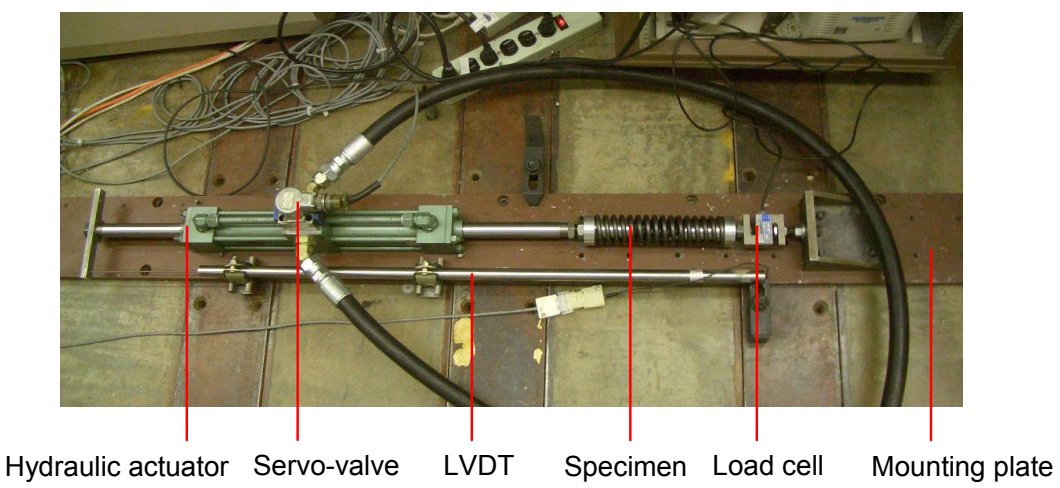

Figure 3. Experimental setup.

This equipment is located at the Smart Structures Technology Laboratory (SSTL) at the University of Illinois at Urbana-Champaign. The load frame is shown in Figure 3. A double-ended hydraulic actuator, manufactured by Nopak, is used. The actuator has a $38.1 \mathrm{~mm}(1.5 \mathrm{in})$ diameter cylinder and a $\pm 152 \mathrm{~mm}(6 \mathrm{in})$ stroke and is equipped with low-friction Teflon seals to reduce nonlinear effects. A Schenck-Pegasus 132A two-stage servo-valve rated for $10 \mathrm{gpm}$ at 1,000 psi pressure drop is used to control the actuator. A pressure accumulator provides supplemental oil flow for high-speed testing. The system is connected to the main hydraulic power supply line of the Newmark Structural Engineering Laboratory operating at 3,000 psi pressure. The actuator is controlled by a SchenckPegasus 5910 digital servo-hydraulic controller in displacement feedback mode. The displacement of the actuator is measured using a Lucas-Schaevitz 10,000 DC-EC linear variable differential transformer (LVDT) having a range of $\pm 254 \mathrm{~mm}$ (10 in). An Omega load cell with a range of $\pm 4,540$ $\mathrm{N}$ (1.0 Kip) is used to measure the applied force. A computer with a dSPACE Real-time control system (DSP) is used to solve the equations of motion and provide real time control. A dSPACE DS2102 High-Resolution 6-channel D/A Board and a dSPACE DS2002/DS2003 32-channel A/D Board are employed to convert the signals from digital to analog, and analog to digital, respectively, both having a resolution of 16 bits. The algorithms (numerical integration and compensation techniques) are implemented in SIMULINK/MATLAB and then downloaded to the dSpace processor using Real-time Workshop software. 

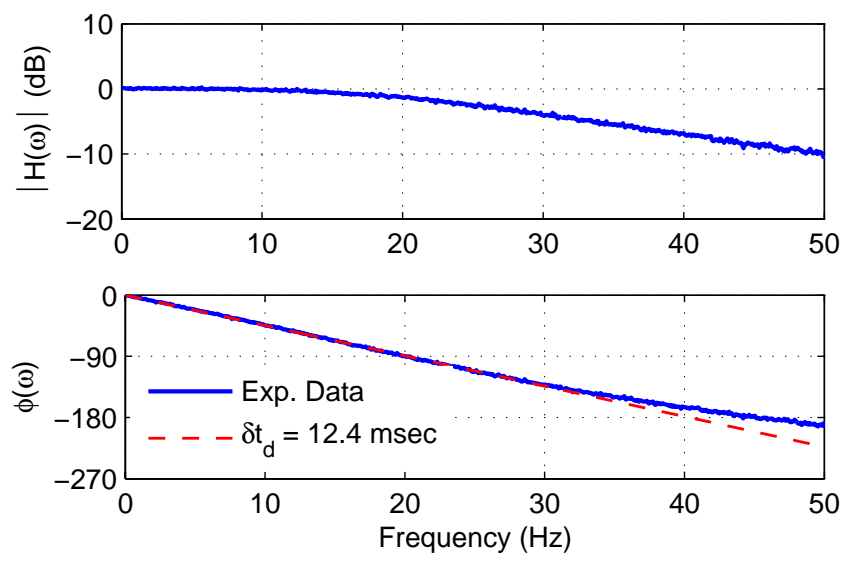

Figure 4. Transfer function from command displacement to measured displacement.

The dynamics of the actuator and the resulting time-lag of the actuator are critical parameters in realtime hybrid testing. To understand the behavior of the system to a wide range of frequencies, the frequency response function from the command displacement to the measured displacement was determined using random excitation. Figure 4 shows the magnitude and phase of the transfer function. After about $15 \mathrm{~Hz}$ the magnitude starts to roll-off and assuming a cutoff frequency of $-3 \mathrm{~dB}$, the bandwidth of the system is determined to be $28.7 \mathrm{~Hz}$. The phase plot shows that within the bandwidth, the phase of the system is very nearly linear. From this slope, the time lag of the actuator is approximated as a time delay of $12.4 \mathrm{msec}$.

\section{VERIFICATION EXPERIMENTS}

Real-time hybrid testing of a simple structural system under earthquake loads was conducted to verify the efficacy of the testing system and compensation strategy. The test structure consisted of a onestory one-bay steel frame assumed to have a rigid beam (i.e., shear frame) which is shown schematically in Figure 5. The structure was divided into two substructures. The left column is tested experimentally (physical substructure) while the right column is modeled numerically (numerical substructure). The columns have stiffness of $3.61 \mathrm{KN} / \mathrm{mm}(20.6 \mathrm{Kip} / \mathrm{in})$ and $6.65 \mathrm{KN} / \mathrm{mm}$ (38.0 Kip/in) for the physical and numerical substructures, respectively. The force-displacement relationship corresponding to the numerical substructure is modeled using a bilinear hysteretic model with a yield displacement of $1 \mathrm{~mm}(0.04 \mathrm{in})$ and a ratio of post- to pre-yield stiffness of 0.02 . Because the purpose of this experiment is to verify the testing system and methodology, instead of using an actual steel cantilever column as the physical substructure, an alternative small-scale specimen was used. The specimen was designed to minimize backlash effects and deformation of the loading frame while fitting into the experimental setup. The specimen is composed of a steel compression spring having a length of $203 \mathrm{~mm}$ ( $8 \mathrm{in})$ and an outside diameter of $49.2 \mathrm{~mm}$ (1.9375 in). The spring has a stiffness of $41.0 \mathrm{~N} / \mathrm{mm}(234 \mathrm{lb} / \mathrm{in})$ therefore a force scaling factor of 88.0 is used. The linear elastic behavior of the spring specimen allows repeatable test results which can be compared against the theoretical expected response, providing an assessment of the effectiveness of the proposed approach.

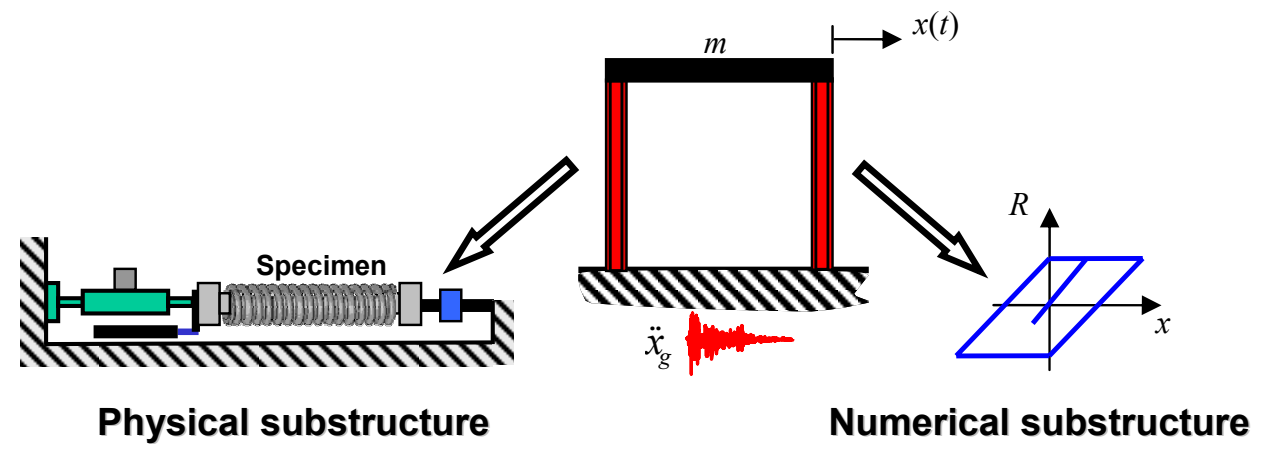

Figure 5. Structural model and substructures for hybrid experiment. 
Several tests were conducted by changing the fundamental frequency of the test structure. During each experiment the mass of the structure was adjusted accordingly to obtain the desired natural frequency. Modal damping was assumed to be $2 \%$ of critical damping. The structure was subjected to the N-S component of the 1940 El Centro earthquake. The Central Difference Method was used for the integration of the equation of motion with a time step $\Delta t=0.0062 \mathrm{sec}$ which corresponds to half of the time delay (i.e., $\delta t_{\mathrm{d}}=2 \Delta t$ ). This time step was adequate to accurately integrate the equation of motion for all the natural frequencies considered in the experiments. The stability criterion for the CDM was easily satisfied with this time step. To evaluate the tests results, a numerical model of the test structure using the experimentally measured stiffness of the test specimen was used.

For the initial set of experiments, the structure was assumed to be linear-elastic; therefore the ratio of post- to pre-yield stiffness in the numerical substructure was set to 1.0. Tests corresponding to natural frequencies of the test structure of $0.5,1,5,6,7,8,10,13$, and $15 \mathrm{~Hz}$ were conducted. The amplitude of the ground acceleration was scaled so that the maximum displacement (calculated using the analytical model) was about $5.08 \mathrm{~mm}(0.2 \mathrm{in})$. For each natural frequency, tests were conducted using (a) no delay compensation, (b) proposed model-based compensation, and (c) third-order polynomial extrapolation. Figure 6 shows the test results for the structure with $1.0 \mathrm{~Hz}$ natural frequency. The duration of the earthquake record used is $15 \mathrm{sec}$, therefore the additional $5 \mathrm{sec}$ correspond to zeros padded at the end of the record to observe the effect of the compensation methods on the free vibration response of the system. As shown in the figure, excellent agreement is obtained between the test results and the analytical solution for both compensation methods (polynomial extrapolation and model-based). The test without delay compensation results in a larger response than the exact analytical solution due to the effect of the negative damping introduced by the uncompensated phase lag from the actuator.

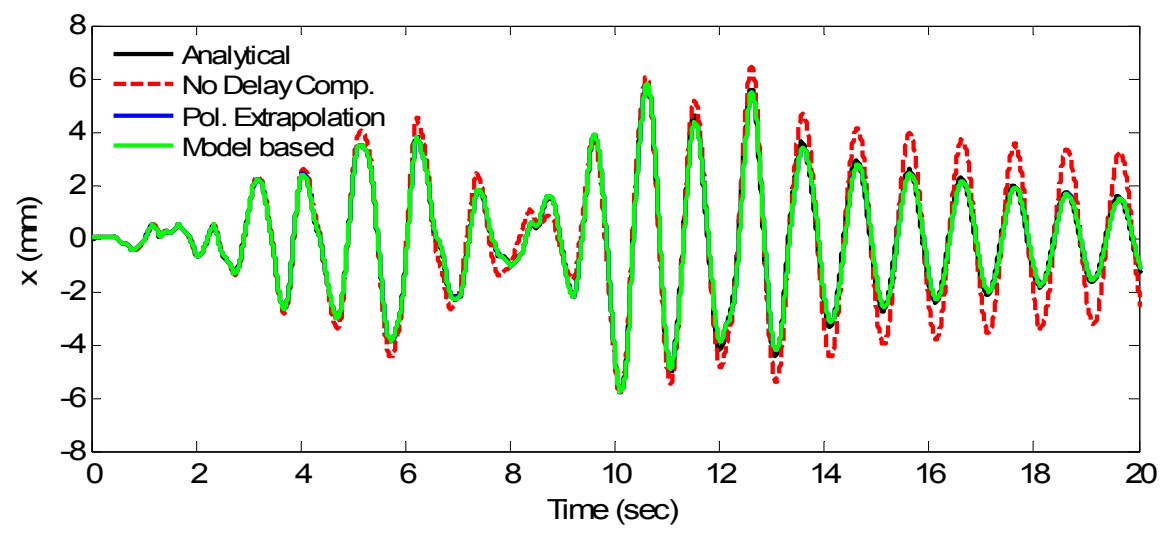

Figure 6. Test results for $1 \mathrm{~Hz}$ natural frequency.

The RMS error between the analytical and experimental results are shown in Figure 7. For each delay compensation method, the highest natural frequency plotted corresponds to the highest natural frequency of the test structure that was tested without the experiment becoming unstable.
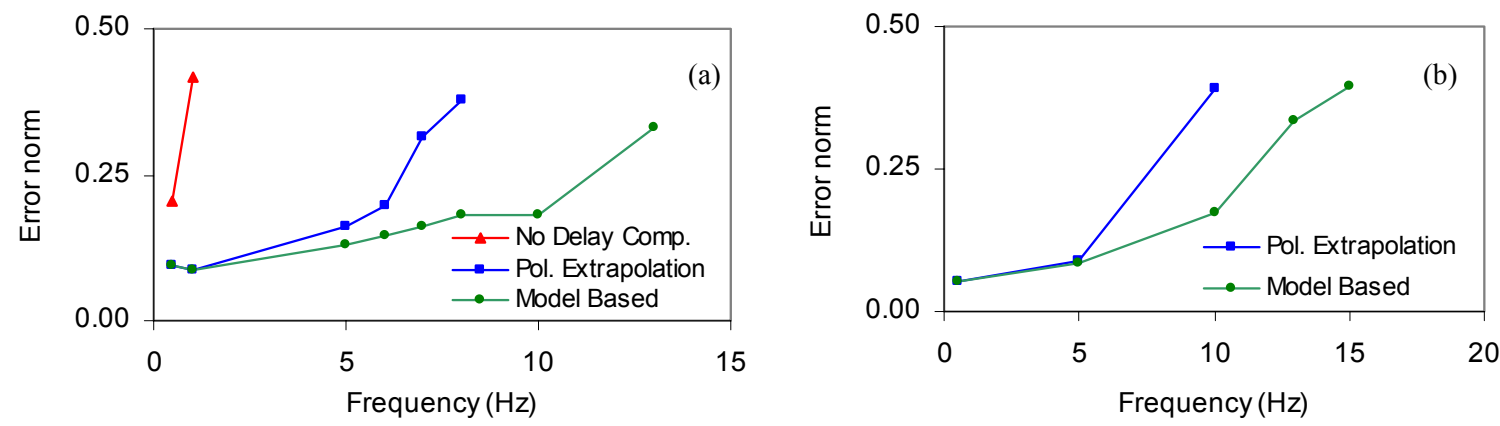

Figure 7. Test results using different delay compensation methods: (a) Linear case, (b) Inelastic case. 
As observed, when no delay compensation is used the experiment becomes unstable even for relatively low frequencies. The theoretical stability limit corresponds to the frequency where the negative damping introduced by the actuator lag becomes equal to the inherent structural damping (1.46 Hz for the test structure considered). Using the proposed model-based compensation method, experiments with natural frequencies as high as $13 \mathrm{~Hz}$ were successfully conducted. The maximum frequency that was effectively tested using the polynomial extrapolation was $8 \mathrm{~Hz}$. Figure 8 shows the results for the test corresponding to a natural frequency of $10 \mathrm{~Hz}$ using model-based delay compensation, where results are seen to match well with the analytical solution.
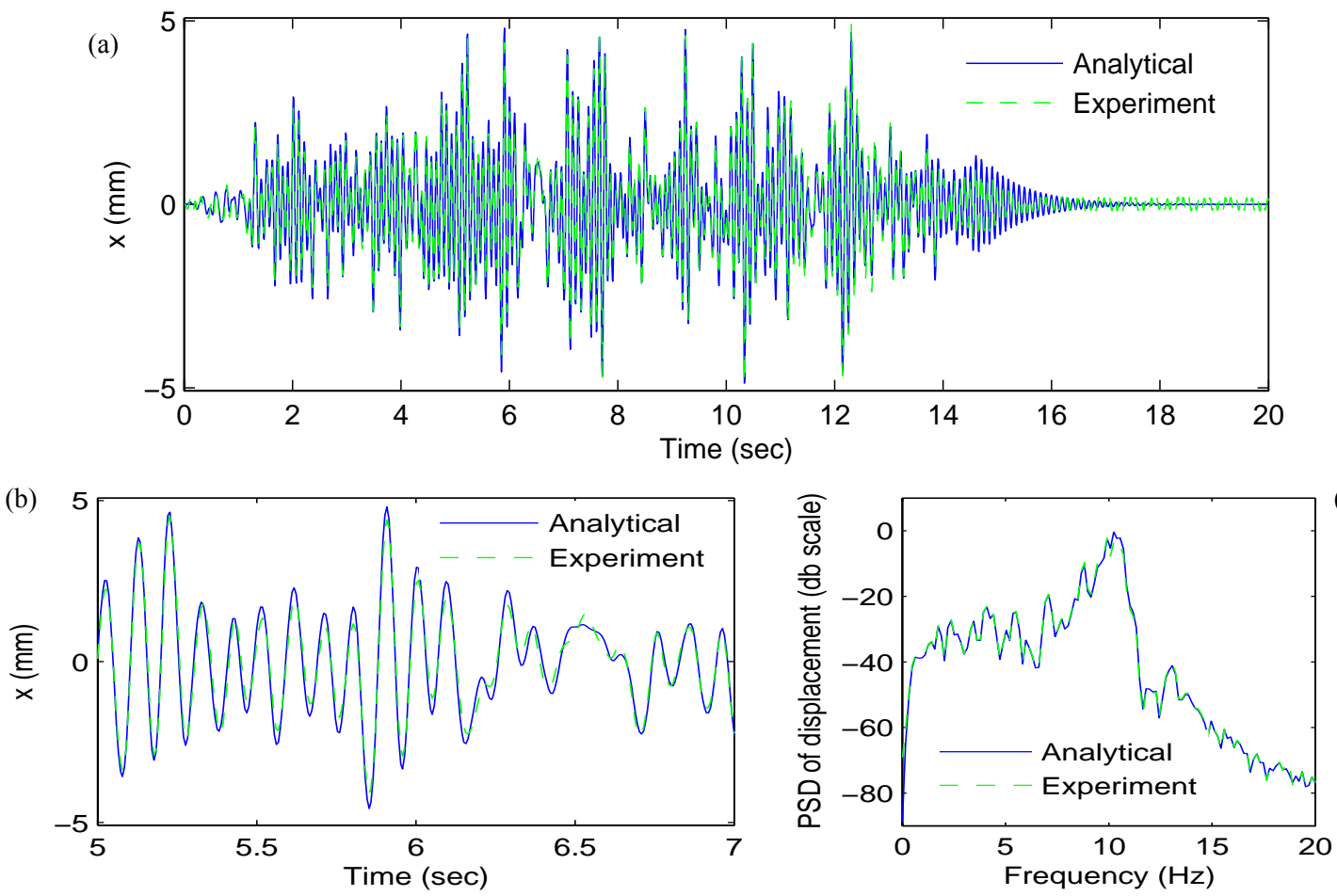

Figure 8. Test results for $10 \mathrm{~Hz}$ natural frequency using model-based compensation: (a) Displacement response, (b) Displacement response (close up view), (c) Power spectrum of displacement.

The maximum natural frequencies for which the experiment was stable using both delay compensation methods are smaller than theoretically predicted. Possible causes are: the effect of experimental errors present in the hybrid experiment, which tend to exacerbate responses at higher frequencies; differences in the estimated actuator time delay and the actual phase lag; the fact that although the phase lag is close to a constant time delay it still has some variations; and the effect of the roll-off in the magnitude of the actuator transfer function (e.g., at a frequency of $15 \mathrm{~Hz}$ the magnitude is 0.94 , causing an undershoot error which adds energy to the system and results in a negative damping which can led to system instability, Mahin et al. 1989).

To evaluate the performance of the delay compensation methods for nonlinear structures, tests were conducted considering a test structure with inelastic response (numerical substructure with bilinear force-displacement relationship). Tests corresponding to natural frequencies of the structure of $0.5,5$, 10,13 , and $15 \mathrm{~Hz}$ were performed using both delay compensation methods. For the model-based compensation method, the initial elastic stiffness was used to approximate the restoring force (Eq. 3). The ratio between maximum displacement and yield displacement (i.e., displacement ductility) was about 4.0. The error norms for the test results are shown on Figure 7(b). As can be seen, the trends are similar to the linear case, however the maximum frequencies that were achieved are slightly larger (15 $\mathrm{Hz}$ for model-based compensation and $10 \mathrm{~Hz}$ for the polynomial extrapolation). This greater stability is due to the damping introduced by the energy dissipated during inelastic deformation. 


\section{CONCLUSIONS}

An approach for real-time hybrid testing has been presented in which time delay/lag compensation is implemented using model-based response prediction. Experimental results demonstrate that the proposed approach performs well for both elastic and inelastic response. Model-based compensation allows testing systems with natural frequencies as high as $13 \mathrm{~Hz}$ for linear response and $15 \mathrm{~Hz}$ for inelastic response, which corresponds to a relatively large bandwidth for hybrid testing. The modelbased compensation method allows testing systems with larger natural frequencies than the traditionally-used polynomial extrapolation. However, the computationally cost is somewhat larger for the model-based approach. Although the specimen and actuator used in this study are of small scale and load capacity, the approach is applicable to larger load capacity actuator and tests specimens.

\section{ACKNOWLEDGMENTS}

This research described in the paper is supported in part by the National Science Foundation under the George E. Brown, Jr. Network for Earthquake Engineering Simulation (NEES) Program (NSF Award No. CMS-0217325).

\section{REFERENCES}

Broyden, C.G. (1965). "A class of methods for solving nonlinear simultaneous equations," Mathematics of Computation, 19, 577-593.

Darby, A.P., Blakeborough, A., and Williams, M.S. (2001). "Improved control algorithm for real-time substructure testing," Earthquake Engineering and Structural Dynamics, 30(3), 431-448.

Dyke, S.J., Spencer, B.F., Quast, P., Sain, M.K. (1995). "Role of control-structure interaction in protective system design," Journal of Engineering Mechanics, ASCE, 121(2), 322-338.

Horiuchi, T., Nakagawa, M., Sugano, M. and Konno, T. (1996). "Development of a real-time hybrid experimental system with actuator delay compensation," In Proc. 11th World Conf. Earthquake Engineering, Paper No. 660.

Horiuchi, T., Inoue, M., Konno, T., and Namita Y. (1999). "Real-time hybrid experimental system with actuator delay compensation and its application to a piping system with energy absorber," Earthquake Engineering and Structural Dynamics, 28(10), 1121-1141.

Mahin, S.A., Shing, P.B., Thewalt, C.R., and Hanson, R.D. (1989) "Pseudodynamic test method. Current status and future directions," Journal of Structural Engineering, ASCE, 115(8), 2113-2128.

Nakashima, M., Kaminosono, T., Ishida, I. and Ando, K. (1990). "Integration techniques for substructure pseudo dynamic test," Proceedings, Fourth U.S. National Conference on Earthquake Engineering, (Volume 2), EERI, Palm Springs, California.

Nakashima, M., Kato, H., and Takaoka, E. (1992). "Development of Real-Time Pseudo Dynamic Testing," Earthquake Engineering and Structural Dynamics, 21(1), 79-92.

Nakashima, M. and Masaoka, N. (1999). "Real Time on-Line Test for MDOF Systems," Earthquake Engineering and Structural Dynamics, 28(4), 393-420.

Shing, P.B., Wei, Z., Jung, R.Y., Stauffer, E. (2004). "Nees Fast Hybrid Test System at the University of Colorado," Proceedings of the 13th World Conference on Earthquake Engineering, Vancouver, Canada, Paper No. 3497.

Takanashi, K., Udagawa, K., Seki, M., Okada, T., and Tanaka H. (1975). "Nonlinear earthquake response analysis of structures by a computer-actuator on-line system," Bulletin of Earthquake Resistant Structure Research Center 8, Institute of Industrial Science, University of Tokyo, Tokio, Japan.

Zhao, J., French, C., Shield, C., Posbergh, T. (2003). "Considerations for the development of real-time dynamic testing using servo-hydraulic actuation," Earthquake Engineering and Structural Dynamics, 32(11), 17731794. 